\title{
Uniform asymptotic solutions of the Cauchy problem for a generalized model equation of $L$. S. Pontryagin in the case of violation of conditions of asymptotic stability
}

\section{Dilmurat Tursunov \\ Department of Algebra and Geometry, Faculty of Mathematics and Information Technology, Osh State University, Osh City, Country Kyrgyzstan}

\section{Email address:}

d_osh@rambler.ru

\section{To cite this article:}

Dilmurat Tursunov. Uniform Asymptotic Solutions of the Cauchy Problem for a Generalized Model Equation of L.S.Pontryagin in the Case of Violation of Conditions of Asymptotic Stability. Science Journal of Applied Mathematics and Statistics. Vol. 1, No. 3, 2013, pp. $25-29$. doi: $10.11648 /$ j.sjams.20130103.11

\begin{abstract}
Here, we construct a uniform asymptotic solution of the Cauchy problem of the small parameter for the inhomogeneous differential equation with small parameter at the derivative, when the linear part of the equation is a pure complex, with its real part changes from negative to positive when one going from the left half to the right half plane.
\end{abstract}

Keywords: Uniform Asymptotic Solution, the Cauchy Problem, the Small Parameter, Inhomogeneous Differential Equation, Model Equation of L. S. Pontryagin

\section{Introduction}

In this paper, we consider a linear, inhomogeneous, ordinary differential equation (ODE) of the first order with a small parameter in the derivative in which the equilibrium position when changing the parameters of the system loses its stability. Models with the change of stability occur in various problems of mechanics, electrical engineering, theory of combustion and explosion, biology and other sciences. It was found that in some cases, by changing the stability of the model can be observed phenomenon of delayed loss of stability.

For the first time the phenomenon of delayed loss of stability for singularly perturbed systems has been described by MA Shishkova [1] student academic Pontryagin. Then, many authors have investigated the nature of this phenomenon. Most full effect of delayed loss of stability for singularly perturbed systems has been described by A.I. Neustadt [2]. And there are works S. Karimov, K.S. Alybaev etc. Using the idea of Professor K. Alymkulov [3], we first construct a uniform asymptotic expansion of the solution of the generalized problem more than the previously cited papers.

\section{Materials and Methods}

Consider the Cauchy problem for a singularly perturbed equation

$$
\begin{gathered}
\varepsilon x^{\prime}(t, \varepsilon)=(t+i) x(t, \varepsilon)+f(t), \\
x(-1, \varepsilon)=x^{0}
\end{gathered}
$$

where $\varepsilon>0$ - small parameter, $f(t)$ - analytic function in $D$, $x^{0}-$ is given constant, $t \in D=\left\{\left(t_{1}, t_{2}\right): t_{1}-\left(t_{2}+1\right) \leq 0, t_{1}+\left(t_{2}+1\right) \geq 0\right.$, $\left.-1 \leq t_{2} \leq 0\right\}, x(t)-$ unknown function.

This problem was first considered by a graduate student [1] of academician L.S.Pontryagin and bounded ness are proved of solution on the interval $t \in[-1,1]$. Here it is constructed a uniform asymptotic expansion of (1)-(2) in $\mathrm{D} \supset[-1,1]$. We will use idea of K.Alymkulov of extract of the singular part for construct asymptotic solution of the singular equation with turning point [3].

The solution of the problem (1)-(2) is sought in the following form

$$
\begin{aligned}
x(\mathrm{t}, \mathcal{\varepsilon})= & \pi_{-1}(\tau, \mu) \mu^{-1}+u_{0}(t)+\pi_{0}(\tau, \mu)+\left(u_{1}(t)+\pi_{1}(\tau, \mu)\right) \mu+\ldots+\left(u_{\mathrm{n}}(t)\right. \\
& \left.+\pi_{n}(\tau, \mu)\right) \mu^{n}+\ldots
\end{aligned}
$$

here $t+i=\tau \mu, \varepsilon=\mu^{2}, u_{\mathrm{n}}(t) \in C^{\infty}(D), n=0,1, \ldots$.

Initial date for $\pi_{k}(\tau, \mu),(k=-1,0,1, \ldots)$ we well take next view: 


$$
\begin{gathered}
\pi_{-1}((-1+i) / \mu, \mu)=\mu\left(x^{0}-u_{0}(-1)\right) ; \\
\pi_{0}((-1+i) / \mu, \mu)=0 ; \\
\pi_{2 \mathrm{n}-1}((-1+i) / \mu, \mu)=0, n \in N ; \\
\pi_{2 \mathrm{n}}((-1+i) / \mu, \mu)=-u_{2 n}(-1), n \in N ;
\end{gathered}
$$

Inserting (3) in (1) we have:

$$
\begin{gathered}
\pi_{-1}^{\prime}(\tau, \mu)+\mu^{2} u_{0}(t)+\mu \pi_{0}^{\prime}(\tau, \mu)+\mu^{3} u_{1}^{\prime}(t)+\mu^{2} \pi_{1}^{\prime}(\tau, \mu)+\ldots= \\
=\tau \pi_{-1}(\tau, \mu)+(t+i) u_{0}(t)+\mu \tau \pi_{0}(\tau, \mu)+\mu(t+i) u_{1}(t)+ \\
+\mu^{2} \tau \pi_{1}(\tau, \mu)+\ldots+f(t)-\sum_{k=0}^{\infty} c_{k} \varepsilon^{k}+\sum_{k=0}^{\infty} c_{k} \mu^{2 k},
\end{gathered}
$$

Here we do not exchange this equation the right part of equality subtracting and adding $\sum_{k=0}^{\infty} c_{k} \mu^{2 k}$. Coefficients $c_{k}$ we will define below.

We have equation for $u_{\mathrm{n}}(t), \pi_{\mathrm{k}}(\tau, \mu)$ :

$$
\begin{gathered}
(t+i) u_{0}(t)+f(t)-c_{0}=0, \\
\pi_{-1}^{\prime}=\tau \pi_{-1}+c_{0}, \\
(t+i) u_{1}(t)=0, \\
u_{2(n-1)}^{\prime}(t)=(t+i) u_{2 n}(t)-c_{n}=0, \\
u_{2 n-1}^{\prime}(t)=(t+i) u_{2 n+1}(t), \\
\pi_{2 n-1}^{\prime}=\tau \pi_{2 n-1}+c_{n}, n \in N . \\
\pi_{0}^{\prime}(\tau)=\tau \pi_{0}(\tau), \\
\pi_{2 n}^{\prime}(\tau)=\tau \pi_{2 n}(\tau), \quad n \in N .
\end{gathered}
$$

From (8) and (11) correspond receive:

$$
\begin{aligned}
& u_{0}(t)=-\frac{f(t)-c_{0}}{t+i}, \text { let } c_{0}=f(-i), \\
& u_{2 n}(t)=\frac{u_{2(n-1)}^{\prime}(t)+c_{n}}{t+i}, \text { let } c_{n}=-u_{2(n-1)}^{\prime}(-i),
\end{aligned}
$$

then

$$
u_{0}(t)=-\left(f_{1}+f_{2}(t+i a)+f_{3}(t+i a)^{2}+\ldots+f_{n}(t+i a)^{n-1}+\ldots\right),
$$$$
\text { here } f_{k}=\frac{1}{k !} \frac{d^{(k)} f(-i)}{d t^{k}}
$$$$
u_{2}(t)=-\left(2 f_{3}+3 f_{4}(t+i a)+4 f_{5}(t+i a)^{2}+\ldots+(n-1) f_{n}(t+i a)^{n-3}+\ldots\right) \text {, }
$$$$
u_{4}(t)=-\left(8 f_{5}+15 f_{6}(t+i a)+24 f_{7}(t+i a)^{2}+\ldots+\right.
$$$$
\left.+(n-1)(n-3) f_{n}(t+i a)^{n-5}+\ldots\right),
$$$$
u_{6}(t)=-\left(48 f_{7}+105 f_{8}(t+i a)+192 f_{9}(t+i a)^{2}+\ldots+\right.
$$

$\left.+(n-1)(n-3)(n-5) f_{n}(t+i a)^{n-7}+\ldots\right)$,

$u_{2 n}(t)=-\left((2 n) ! ! f_{2 n+1}+(2 n+1) ! ! f_{2 n+2}(t+i a)+\ldots+\right.$

$\left.+(k-1)(k-3) \ldots(k-2 n+1) f_{n}(t+i a)^{n-2 \mathrm{n}-1}+\ldots\right), \quad k \geq 2 n+1$.

$c_{1}=f_{2} ; \quad c_{2}=3 f_{4} ; \quad c_{3}=5 * 3 f_{6} ; \quad c_{4}=7 * 5 * 3 f_{8} ; \quad c_{n}=(2 n-1) ! ! f_{2 n}$.

From (10) and (12) have $u_{2 n-1}(t) \equiv 0, n \in N$.

Then $u_{\mathrm{n}}(t) \in C^{\infty}(D), n=0,1, \ldots$.

Solutions of problem (9)-(4), (14)-(5), (13)-(6), (15)-(7) will represent in the view:

$$
\pi_{-1}(\tau)=\mu\left(x^{0}-u_{0}(-1)\right) e^{\frac{\mu^{2} \tau^{2}+2 i}{2 \mu^{2}}}+e^{\frac{\tau^{2}}{2}} c_{0} \int_{\frac{-1+i}{\mu}}^{\tau} e^{-\frac{s^{2}}{2}} d s
$$

$$
\pi_{2 n-1}(\tau)=e^{\frac{\tau^{2}}{2}} c_{n} \int_{\frac{-1+i}{\mu}}^{\tau} e^{-\frac{s^{2}}{2}} d s, n \in N
$$

$\pi_{2 n}(\tau, \mu)=-u_{2 n}(-1) e^{\frac{\mu^{2} \tau^{2}+2 i}{2 \mu^{2}}}, n \in N$

$\pi_{0}(\tau, \mu) \equiv 0$.

We have

$$
\begin{aligned}
& \pi_{2 n-1}(\tau)=-\frac{c_{n}}{\tau}\left(1-\frac{1}{\tau^{2}}+\frac{3}{\tau^{4}}-\ldots(-1)^{k} \frac{(2 k-1) ! !}{\tau^{2 k}}+\ldots\right), \\
& \pi_{2 n}(\tau)=0, n=0,1, \ldots, \quad \tau \rightarrow \infty
\end{aligned}
$$

Lemma. For the integral

$$
J(\tau, \mu)=e^{\tau^{2} / 2} \int_{(-1+i) / \mu}^{\tau} e^{-s^{2} / 2} d s
$$

here $\tau=(t+i) / \mu$.

We have estimate

$$
|J(t, \mu)|=\left\{\begin{array}{l}
O(\mu), \text { if } t \in D_{0} \\
O(1), \text { if } t \in D_{\mu}
\end{array}\right.
$$

here $t=t_{1}+i t_{2}, D_{0}=\left\{\left(t_{1} ; t_{2}\right): t_{1}+\left(t_{2}+1\right) \geq 0, t_{1}-\left(t_{2}+1\right) \leq-\mu, t_{2} \leq 0\right\}$, $D_{\mu}=\left\{\left(t_{1} ; t_{2}\right): t^{2}{ }_{1}-\left(t_{2}+1\right)^{2} \leq 0,-1 \leq t_{2} \leq 0, t_{1}-\left(t_{2}+1\right) \geq-\mu\right\}$.

Proof. If we turn to variable $t$ then $J(t, \mu)=e^{\frac{(t+i)^{2}}{2 \mu^{2}}} \int_{(-1+i) / \mu}^{(t+i) / \mu} e^{-s^{2} / 2} d s=\frac{1}{\mu} e^{\frac{(t+i)^{2}}{2 \mu^{2}}} \int_{-1}^{t} e^{-\frac{(\xi+i)^{2}}{2 \mu^{2}}} d \xi$,

Let $\xi=\xi_{1}+i \xi_{2}$, then 


$$
J\left(t_{1}, t_{2}, \mu\right)=\frac{1}{\mu} e^{\frac{\left(t_{1}+i\left(t_{2}+1\right)\right)^{2}}{2 \mu^{2}}} \int_{L} e^{-\frac{\left(\xi_{1}+i\left(\xi_{2}+1\right)\right)^{2}}{2 \mu^{2}}} d\left(\xi_{1}+i \xi_{2}\right) .
$$

We consider following cases:

1) If $\left(t_{1}, t_{2}\right) \in D \cap\left(t_{1} \leq 0\right)$ then way of integrating $L$ consist of segments $\mathrm{L}_{1}$ and $\mathrm{L}_{2}$, where $\mathrm{L}_{1}: \xi_{1}+\left(\xi_{2}+1\right)=0,-1 \leq \xi_{1} \leq 0 ; \mathrm{L}_{2}$ : $\xi_{1}=t_{1},-1-\mathrm{t}_{1} \leq \xi_{2} \leq t_{2}$.

2) If $\left(t_{1}, t_{2}\right) \in D \cap\left(t_{1} \geq 0\right)$ then way of integrating $L$ consist of segments $\mathrm{L}_{1}, \mathrm{~L}_{2}$ and $\mathrm{L}_{3}$ where $\mathrm{L}_{1}: \xi_{1}+\left(\xi_{2}+1\right)=0,-1 \leq \xi_{1} \leq 0$; $\mathrm{L}_{2}: \xi_{1}-\left(\xi_{2}+1\right)=0,0 \leq \xi_{1} \leq t_{1} ; \mathrm{L}_{3}: \xi_{1}=t_{1}, t_{1}-1 \leq \xi_{2} \leq t_{2}$.

In the first case

$$
J\left(t_{1}, t_{2}, \mu\right)=\frac{1}{\mu} K\left(t_{1}, t_{2}, \varepsilon\right)\left(\int_{-1}^{t_{1}} e^{\frac{i \xi_{1}^{2}}{\mu^{2}}} d \xi_{1}+\int_{-1-t_{1}}^{t_{2}} e^{-\frac{\left(t_{1}+i\left(\xi_{2}+1\right)\right)^{2}}{2 \mu^{2}}} d \xi_{2}\right),
$$

In the second case

$J\left(t_{1}, t_{2}, \mu\right)=\frac{1}{\mu} K\left(t_{1}, t_{2}, \varepsilon\right)\left(\int_{-1}^{0} e^{\frac{i \xi_{1}^{2}}{\mu^{2}}} d \xi_{1}+\int_{0}^{t_{1}} e^{-\frac{i \xi_{1}^{2}}{\mu^{2}}} d \xi_{1}+\int_{-1+t_{1}}^{t_{2}} e^{-\frac{\left(t_{1}+i\left(\xi_{2}+1\right)\right)^{2}}{2 \mu^{2}}} d \xi_{2}\right)$,

here $K\left(t_{1}, t_{2}, \varepsilon\right)=\exp \left(\frac{\left(t_{1}+i\left(t_{2}+i\right)\right)^{2}}{2 \mu^{2}}\right)$.

In (17) integrating by parts we have:

$$
\left|\int_{-1}^{t_{1}} e^{\frac{i \xi_{1}^{2}}{\mu^{2}}} d \xi_{1}\right| \leq c \mu^{2}
$$

if $-\delta<t_{1} \leq 0, \delta>0$ - sufficiently small, and by applying the stationary phase method we obtain

$$
\left|\int_{-1}^{t_{1}} e^{\frac{i \xi_{1}^{2}}{\mu^{2}}} d \xi_{1}\right| \leq c \mu, \quad c>0-\text { const. }
$$

Analogously in (18), we have

$$
\left|\int_{-1}^{0} e^{\frac{i \xi_{1}^{2}}{\mu^{2}}} d \xi_{1}+\int_{0}^{t_{1}} e^{-\frac{i \xi_{1}^{2}}{\mu^{2}}} d \xi_{1}\right| \leq c \mu .
$$

If we set $k_{1}=K\left(t_{1}, t_{2}, \varepsilon\right) \int_{-1+t_{1}}^{t_{2}} e^{-\frac{\left(t_{1}+i\left(\xi_{2}+1\right)\right)^{2}}{2 \mu^{2}}} d \xi_{2}$, then

$\left|k_{1}\right| \leq \int_{-1+t_{1}}^{t_{2}} e^{-\frac{\left(t_{2}-\tau_{2}\right)\left(t_{2}+\tau_{2}+2\right)}{2 \mu^{2}}} d \tau_{2} \leq \int_{-1+t_{1}}^{t_{2}} e^{-\frac{\left(t_{2}-\tau_{2}\right)\left(t_{2}+t_{1}+1\right)}{2 \mu^{2}}} d \tau_{2}=$

$=\frac{2 \mu^{2}}{1+t_{1}+t_{2}}\left(1-e^{\frac{t_{1}^{2}-\left(t_{2}+1\right)^{2}}{2 \mu^{2}}}\right)$

Thus, we is proved (16).
Consequently, series (3) is asymptotic in domain $D$.

Series (3) is asymptotical series, that is

$x(t, \mu)=\frac{\pi_{-1}(\tau)}{\mu}+\sum_{k=0}^{n} \mu^{k}\left(u_{k}(t)+\pi_{k}(\tau, \mu)\right)+$

$+\mu^{n+1} R_{n+1}(t, \mu)$,

where $\left|R_{n+1}(t, \mu)\right| \leq m=$ const . Realy for $R_{n+1}(t, \mu)$ we have next problem:

$$
\mu^{2} R_{n+1}^{\prime}(t, \mu)=(t+i) R_{n+1}(t, \mu)-\mu u_{n}^{\prime}(t),
$$

$$
R(-1)=0 \text {. }
$$

From here

$$
R_{n+1}(t, \mu)=-\frac{1}{\mu} \int_{-1}^{t} u_{n}^{\prime}(\tau, \varepsilon) e^{\frac{(t+i)^{2}-(\tau+i)^{2}}{2 \varepsilon}} d \tau .
$$

From lemma 1 follow next estimate

$$
\left|R_{n+1}(t, \mu)\right|=\left\{\begin{array}{l}
O(\mu), \text { if } t \in D_{0}, \\
O(1), \text { if } t \in D_{\mu} .
\end{array}\right.
$$

Theorem. Problem (1)-(2) has a unique solution and satisfies the asymptotic expansion (3).

The expansion (3) can be obtained in another way, i.e. without going into the complex plane.

We write the solution of the problem (1)-(2) in the integral form:

$x(t, \varepsilon)=x^{0} e^{\frac{(t+i)^{2}-\left(t_{0}+i\right)^{2}}{2 \varepsilon}}+\frac{1}{\varepsilon} \int_{-1}^{t} e^{\frac{(t+i)^{2}-(\tau+i)^{2}}{2 \varepsilon}} f(\tau) d \tau$

$$
\text { Let } f(t)=\sum_{k=0}^{\infty} f_{k}(t+i)^{k}, f_{k}=\frac{1}{k !} \frac{d^{(k)} f(-i)}{d t^{k}} \text {, then }
$$

$x(t, \varepsilon)=x^{0} e^{\frac{(t+i)^{2}-\left(t_{0}+i\right)^{2}}{2 \varepsilon}}+\frac{1}{\varepsilon} \sum_{k=0}^{\infty} f_{k} I_{k}(t, \varepsilon)$

where $I_{k}(t, \varepsilon)=\int_{-1}^{t}(\tau+i)^{k} e^{\frac{(t+i)^{2}-(\tau+i)^{2}}{2 \varepsilon}} d \tau$

$$
\text { If } I_{0}(t, \varepsilon)=\int_{-1}^{t} e^{\frac{(t+i)^{2}-(\tau+i)^{2}}{2 \varepsilon}} d \tau
$$

parameter $\varepsilon$, we obtain:

differentiate the smal

$$
I_{2}(t, \varepsilon)=2 \varepsilon^{2} \frac{\partial I_{0}(t, \varepsilon)}{\partial \varepsilon}+(t+i)^{2} I_{0}(t, \varepsilon)
$$

this implies 


$$
\begin{aligned}
& I_{4}(t, \varepsilon)=2 \varepsilon^{2} \frac{\partial I_{2}(t, \varepsilon)}{\partial \varepsilon}+(t+i)^{2} I_{2}(t, \varepsilon) \\
& I_{6}(t, \varepsilon)=2 \varepsilon^{2} \frac{\partial I_{4}(t, \varepsilon)}{\partial \varepsilon}+(t+i)^{2} I_{4}(t, \varepsilon) \\
& I_{2 n}(t, \varepsilon)=2 \varepsilon^{2} \frac{\partial I_{2(n-1)}(t, \varepsilon)}{\partial \varepsilon}+(t+i)^{2} I_{2(n-1)}(t, \varepsilon)
\end{aligned}
$$

For the odd indices:

$$
\begin{aligned}
& I_{3}(t, \varepsilon)=2 \varepsilon^{2} \frac{\partial I_{1}(t, \varepsilon)}{\partial \varepsilon}+(t+i)^{2} I_{1}(t, \varepsilon) ; \\
& I_{5}(t, \varepsilon)=2 \varepsilon^{2} \frac{\partial I_{3}(t, \varepsilon)}{\partial \varepsilon}+(t+i)^{2} I_{3}(t, \varepsilon) ; \\
& I_{2 n+1}(t, \varepsilon)=2 \varepsilon^{2} \frac{\partial I_{2 n-1}(t, \varepsilon)}{\partial \varepsilon}+(t+i)^{2} I_{2 n-1}(t, \varepsilon) ;
\end{aligned}
$$$$
n \in \mathrm{N},
$$

where

$$
\begin{aligned}
& I_{1}(t, \varepsilon)=\int_{-1}^{t}(t+i) e^{\frac{(t+i)^{2}-(\tau+i)^{2}}{2 \varepsilon}} d \tau= \\
& =\varepsilon\left(e^{\frac{(t+i)^{2}-\left(t_{0}+i\right)^{2}}{2 \varepsilon}}-1\right)=O(\varepsilon) .
\end{aligned}
$$

From Lemma for $I_{0}(t, \varepsilon)$ we obtain:

$$
\text { . }\left|I_{0}(t, \mu)\right|=\left\{\begin{array}{l}
O\left(\mu^{2}\right), \text { при } t \in D_{0} \\
O(\mu), \text { при } t \in D_{\mu}
\end{array}\right.
$$

Therefore

$$
x(t, \varepsilon)=\left\{\begin{array}{l}
x^{0} e^{\frac{(t+i)^{2}-\left(t_{0}+i\right)^{2}}{2 \varepsilon}}+\frac{1}{\varepsilon} \sum_{k=0}^{\infty} f_{k} c_{k} \varepsilon^{(k+1)}, \text { if } t \in D_{0}, \\
x^{0} e^{\frac{(t+i)^{2}-\left(t_{0}+i\right)^{2}}{2 \varepsilon}}+\frac{1}{\varepsilon} \sum_{k=0}^{\infty} f_{k} c_{k} \varepsilon^{(k+1) / 2}, \text { if } t \in D_{\mu} .
\end{array}\right.
$$

it is asymptotically equivalent to the asymptotic series (3).

The integral $I_{0}(t, \varepsilon)=\int_{-1}^{t} e^{\frac{(t+i)^{2}-(\tau+i)^{2}}{2 \varepsilon}} d \tau$ can be estimated without having to go into the complex plane as follows.

$$
\text { Introduce a new parameter } a \text {. }
$$

$I_{0}(t, \varepsilon, a)=\int_{-1}^{t} e^{\frac{(t+i a)^{2}-(\tau+i a)^{2}}{2 \varepsilon}} d \tau$

and divide the integral into two integrals

$$
\begin{aligned}
& I_{0}(t, \varepsilon, a)=\int_{-1}^{t} e^{\frac{(t+i a)^{2}-(\tau+i a)^{2}}{2 \varepsilon}} d \tau= \\
& =\int_{-1}^{-t} e^{\frac{(t+i a)^{2}-(\tau+i a)^{2}}{2 \varepsilon}} d \tau+\int_{-1}^{-t} e^{\frac{(t+i a)^{2}-(\tau+i a)^{2}}{2 \varepsilon}} d \tau .
\end{aligned}
$$

The first integral is estimated by integration by parts:

$$
\begin{aligned}
& \int_{-1}^{-t} e^{\frac{(t+i a)^{2}-(\tau+i a)^{2}}{2 \varepsilon}} d \tau=e^{\frac{(t+i a)^{2}}{2 \varepsilon}} \int_{-1}^{-t} e^{\frac{(\tau+i a)^{2}}{2 \varepsilon}} d \tau= \\
& =e^{\frac{(t+i a)^{2}}{2 \varepsilon}} \int_{-1}^{-t} \frac{-\varepsilon}{\tau+i a} d e^{-\frac{(\tau+i a)^{2}}{2 \varepsilon}}=\varepsilon\left(\frac{1}{a i-1} e^{\frac{(t+i a)^{2}+2 i a^{2}}{2 \varepsilon}}-\right. \\
& \left.-\frac{1}{(i a-t)} e^{\frac{i 2 a t}{\varepsilon}}-\int_{-1}^{-t} \frac{\varepsilon}{(\tau+i a)^{3}} d e^{-\frac{(\tau+i a)^{2}}{2 \varepsilon}}\right)
\end{aligned}
$$

Therefore

$$
\int_{-1}^{-t} e^{\frac{(t+i a)^{2}-(\tau+i a)^{2}}{2 \varepsilon}} d \tau=O(\varepsilon),-t<0
$$

We now estimate the second integral:

$$
\begin{aligned}
& \int_{-t}^{t} e^{\frac{(t+i a)^{2}-(\tau+i a)^{2}}{2 \varepsilon}} d \tau=e^{\frac{(t+i a)^{2}}{2 \varepsilon}} \int_{-t}^{t} e^{-\frac{(\tau+i a)^{2}}{2 \varepsilon}} d \tau= \\
& =e^{\frac{(t+i a)^{2}}{2 \varepsilon}} e^{\frac{a^{2}}{2 \varepsilon}} \int_{-t}^{t} e^{-\frac{\tau^{2}}{2 \varepsilon}+i \frac{a \tau}{\varepsilon}} d \tau= \\
& =e^{\frac{(t+i a)^{2}}{2 \varepsilon}} e^{\frac{a^{2}}{2 \varepsilon}} \int_{-t}^{t} e^{-\frac{\tau^{2}}{2 \varepsilon}}\left(\cos \left(\frac{a \tau}{\varepsilon}\right)-i \sin \left(\frac{a \tau}{\varepsilon}\right)\right) d \tau= \\
& =e^{\frac{(t+i a)^{2}}{2 \varepsilon}} e^{\frac{a^{2}}{2 \varepsilon}} \mathrm{Y}(t, \varepsilon, a) ; \\
& \text { where } \mathrm{Y}(t, \varepsilon, a)=\int_{-t}^{t} e^{-\frac{\tau^{2}}{2 \varepsilon}} \cos \left(\frac{a \tau}{\varepsilon}\right) d \tau .
\end{aligned}
$$

function $\mathrm{Y}(t, \varepsilon, a)$ is differentiable in the parameter $a$ :

$$
\frac{\partial \mathrm{Y}(t, \varepsilon, a)}{\partial a}=-\int_{-t}^{t} \frac{\tau}{\varepsilon} e^{-\frac{\tau^{2}}{2 \varepsilon}} \sin \left(\frac{a \tau}{\varepsilon}\right) d \tau=
$$


$=\left.e^{-\frac{\tau^{2}}{2 \varepsilon}} \sin \left(\frac{a \tau}{\varepsilon}\right)\right|_{-t} ^{t}-\frac{a}{\varepsilon} \int_{-t}^{t} e^{-\frac{\tau^{2}}{2 \varepsilon}} \cos \left(\frac{a \tau}{\varepsilon}\right) d \tau$.

As $e^{-\frac{t^{2}}{2 \varepsilon}} \sin (a \tau) \sim 0, \varepsilon \rightarrow 0$, we have:

$\frac{\partial \mathrm{Y}(t, \varepsilon, a)}{\partial a}=-\frac{a}{\varepsilon} \mathrm{Y}(t, a) \Rightarrow \mathrm{Y}(t, \varepsilon, a)=c e^{-\frac{a^{2}}{2 \varepsilon}}$.

If $a=0$ then we have $\mathrm{Y}(t, \varepsilon, 0)=\int_{-t}^{t} e^{-\frac{\tau^{2}}{2 \varepsilon}} d \tau=$

$=\sqrt{2 \varepsilon} \int_{-t / \sqrt{2 \varepsilon}}^{t / \sqrt{2 \varepsilon}} e^{-z^{2}} d z \sim \sqrt{2 \varepsilon} \int_{-\infty}^{\infty} e^{-z^{2}} d z=\sqrt{2 \varepsilon \pi}$.

On the other hand $\mathrm{Y}(t, \varepsilon, 0)=c$.

Therefore $c=\sqrt{2 \varepsilon \pi}$ and $\mathrm{Y}(t, \varepsilon, a)=\sqrt{2 \varepsilon \pi} e^{-\frac{a^{2}}{2 \varepsilon}}$

$$
\begin{aligned}
& I_{0}(t, \varepsilon, a)=O(\varepsilon)+e^{\frac{(t+i a)^{2}}{2 \varepsilon}} e^{\frac{a^{2}}{2 \varepsilon}} \sqrt{2 \varepsilon \pi e^{-\frac{a^{2}}{2 \varepsilon}}}= \\
& =O(\varepsilon)+\sqrt{2 \varepsilon \pi} e^{\frac{(t+i a)^{2}}{2 \varepsilon}} .
\end{aligned}
$$

Therefore

$$
\left|I_{0}(t, \varepsilon)\right|=O\left(\sqrt{\varepsilon} e^{\frac{t^{2}-1}{2 \varepsilon}}\right) .
$$

Remark. The considered problem may be generalized to the system of differential equations.

$\varepsilon x^{\prime}(t, \varepsilon)=A(t) x(t, \varepsilon)+f(t), \quad x(-1, \varepsilon)=x^{0}$.

Where $A(t)$ - matrix-function of the second order have $\lambda_{1}(t)=t+i, \lambda_{2}(t)=t-i$, eigen values, for example

$$
A(t)=\left(\begin{array}{cc}
t & -1 \\
1 & t
\end{array}\right) \text {. }
$$

\section{References}

[1] Shishkova M.A. Consideration of a system of differential equations with a small parameter in the highest derivatives // DAN AN SSSR, 1973. - V. 209. - № 3. - PP.576-579.

[2] Neishtadt A.I. Spanning the loss of stability for dynamic bifurcations. Dif.eq. 1987, v. 23, 12, -PP.2060-2067

[3] Alymkulov K. Extension of boundary layer function method for singularly perturbed differential equation of Prandtle Tichonov and Lighthill types // Reports of the third congress of the world mathematical society of Turkic countries, Almaty, June July, 2009, -PP 256-259. 